\title{
PSEUDOMYXOMA PERITONEI - AN ENDOSCOPIC IMAGE
}

\section{Shafqat Mehmood ${ }^{1}$, Syed Aamir Ali Shah², Syed Ather Kazmi ${ }^{3}$, Faisal Zeb ${ }^{1}$}

${ }^{1}$ Departments of Internal Medicine, Shaukat Khanum Memorial Cancer Hospital and Research Centre Lahore, Pakistan, ${ }^{2}$ Department of Surgical Oncology, Shaukat Khanum Memorial Cancer Hospital and Research Centre Lahore, Pakistan, ${ }^{3}$ Department of Medical Oncology, Shaukat Khanum Memorial Cancer Hospital and Research Centre Lahore, Pakistan

Received: 9 April 2015 / Accepted: 23 April 2015

A 61-year-old male presented to our department with a history of worsening abdominal distention for the past 1 month. Initial ultrasound abdomen showed debrinous ascites with loculations. A computed tomography (CT) imaging of the chest and abdomen was done with I/V contrast that showed gross peritoneal thickening and omental cake formation with deposits in peritoneum suggestive of pseudomyxoma peritonei. CT-guided biopsy of the omentum was performed which was reported as metastatic mucinous adenocarcinoma of unknown primary.

The patient was planned for upper and lower gastrointestinal (GI) endoscopies to rule out primary tumour in the GI tract. Upper GI endoscopy was normal; however, colonoscopy showed polypoidal growth at the orifice of appendix [Figure 1]. Multiple biopsies were taken. A laparotomy was performed which showed massive ascites with myxomatous fluid and omental cake formation. In addition to this, there was a nodular mass involving the appendix. A limited right hemicolectomy, omentectomy and debulking surgery were performed. Histopathology of the appendix showed mucinous adenocarcinoma. The patient was discharged home after a week with an appointment in medical oncology clinic for adjuvant treatment.

Pseudomyxoma peritonei is a rare disease process characterised by copious amount of mucinous ascites and histologically bland peritoneal mucinous tumour. ${ }^{[1]}$ It is mostly appendicular in origin. The treatment option for this tumour includes repeated abdominal surgeries and cytoreductive approach, which includes intraoperative

Correspondence: Dr. Shafqat Mehmood, Department of Internal Medicine, Shaukat Khanum Memorial Cancer Hospital and Research Centre, Johar Town, Lahore, Pakistan.

Email: smehmood@skm.org.pk

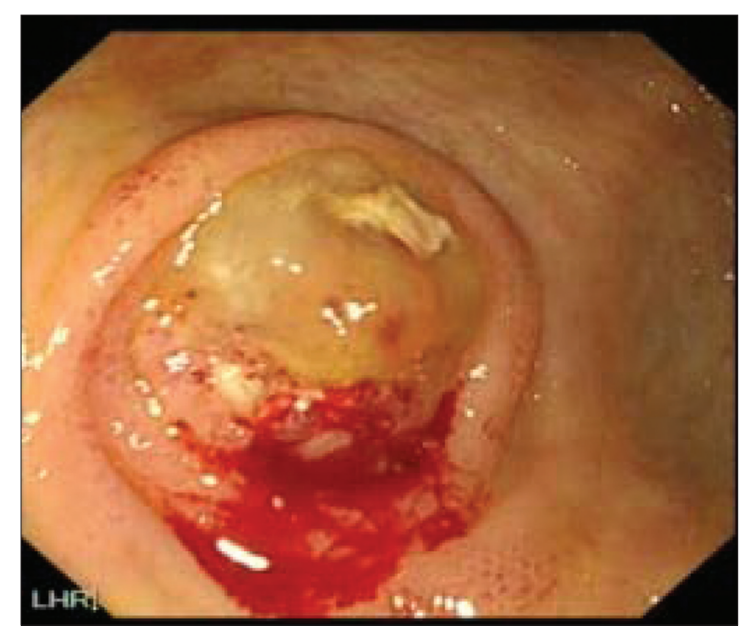

Figure 1: Colonoscopic image showing tumour of the appendix

and perioperative chemotherapy. ${ }^{[2,3]}$ The overall prognosis is guarded and only a small percentage of the patients are alive at 5 years..$^{[1,2]}$ In conclusion, pseudomyxoma peritonei is a rare disease, usually of appendiceal in origin and can be diagnosed effectively on colonoscopy.

\section{Conflict of Interest}

The authors declare that they have no conflict of interest.

\section{References}

1. Miner TJ, Shia J, Jaques DP, et al. Long term survival following treatment in pseudomyxoma peritonei. Ann Surg 2005;241:300-8.

2. Sugarbaker PH. Cytoreductive surgery and peri-operative intraperitoneal chemotherapy as a curative approach to pseudomyxoma peritonei syndrome. Eur J Surg Oncol 2001;27:239-43.

3. Sugarbaker PH, Jablonski KA. Prognostic features of 51 colorectal and 30 appendiceal cancer patients with peritoneal carcinomatosis treated by cytoreductive surgery and intraperitoneal chemotherapy. Ann Surg 1995;221: 